\title{
LOAD MODELING USING ANFIS TOOL IN VOLTAGE STABILITY STUDIES FOR 6 BUS AND 30 BUS SYSTEM
}

\author{
R. SUGANYA, B. BALAJI \& D. GAYATHRI
}

AP/EEE, IFET College of Engineering, Villupuram, Tamil Nadu

\begin{abstract}
Voltage stability continues to be a limiting phenomenon in many power systems. When combined with a continual growth in load, the lack of sufficient and optimally located generation together with the failure to build new transmission facilities has lead many systems to be vulnerable to situations of uncontrollable system voltages. Voltage Instability can result in system blackout. To deal with this serious issue, many utilities have mandated the study of voltage stability as a normal component in system planning and operation. While acceptable methods of voltage stability analysis have emerged in recent years, and comprehensive tools have been developed, the issue of load modeling remains a challenge. This paper is to model the loads in order to predict the maximum deliverable power. Modeling a load is a difficult problem because power system loads are aggregates of different devices. Load modeling is representation of composite loads in computer simulations designed to study load flows and dynamic system characteristics.

In this paper, modeling of voltage dependent load and ZIP load is carried out using normal load modeling and using ANFIS. Error is calculated between the two models. Modal analysis is carried out to determine the weakest bus in the system which gives a chance to improve the stability of the system. Modeling has been carried out using six bus system and thirty bus system for both voltage dependent load and ZIP load. The results of real and reactive power are compared and reported. ANFIS model the system with most accuracy.
\end{abstract}

KEYWORDS: Load Modeling, Voltage Dependent Load Load, Maximum Power \& Voltage Stability

Received: May 13, 2020; Accepted: Jun 03, 2020; Published: Aug 04, 2020; Paper Id.: IJMPERDJUN2020598

\section{INTRODUCTION}

Power system stability is a multi-faceted problem depending upon a variety of factors, the time span that must be taken into consideration in order to assess stability/instability, the size of the disturbance considered, the physical nature of the resulting instability.

Transmission network need to be utilized ever more efficiently. The more efficient use of transmission network has already led to a situation in which many power systems are operated more often and longer close to voltage stability limits. A power system stressed by heavy loading has a different response to disturbances from that of a non-stressed system. The potential size and effect of disturbances has also increased. When a power system is operated closer to stability limit, a relatively small disturbance may cause a system upset. In addition, larger areas of the interconnected system may be affected by a disturbance.

The assessment of voltage stability has become more complicated due to strengthening of power systems. Environmental constraints limit the expansion of a transmission network and generation near load centre which has a negative influence on power system voltage stability, because electrical distance from a generator to a load increases 
and voltage support weakens in the load area. The generation today often takes place in fewer and larger units located at considerable distances from major loads. Simultaneous growth in the use of electrical power without a corresponding increase of transmission capacity has brought many power systems close to voltage stability limits.

Transmission systems are usually reliable due to redundancy in systems. The reliability of transmission systems is determined according to line overload, protection, power system dynamic behaviour. However the loss of a power system stability may cause a total blackout of the system.

\section{VOLTAGE STABILITY ISSUES}

Power system is Stable if voltages after a disturbance are close to voltages at normal operating condition. A Power system becomes unstable when voltages uncontrollably decrease due to outage of equipment (generator, line, transformer, busbar etc), increment in load, decrement of production and weakening of voltage control.

The definition of voltage instability stems from the attempt of load dynamics to restore power consumption beyond the capability of the combined transmission and generation system.

Voltage stability can also be called as load stability. A power system lacks the capability to transfer an infinite amount of electrical power to the loads. The main factor causing voltage instability is the inability of the power system to meet the demands for reactive power in the heavily stressed systems to keep desired voltages. Other factors contributing to voltage stability are the generator reactive power limits, the load characteristics, the characteristics of the reactive power compensation devices and the action of the voltage control devices. The power system lacks the capability to transfer power over long distances. Transfer of reactive power is difficult due to extremely high reactive power losses, which is why the reactive power required for voltage control is produced and consumed at the control area.

In Modern power systems, frequency stability problems can be encountered after a major disturbance has resulted in islanding. Since we have assumed that the electromechanical oscillations have died out, frequency is common throughout each island and the problem can be analyzed using a single bus equivalent on which all generators and loads are connected. The frequency instability is related to the active power imbalance between generators and loads in each island.

Voltage stability requires a full network representation for its analysis. This is a main aspect separating the two classes of long term stability problems. Voltage stability is a load driven problem now referring to voltage stability we can identify dynamic load components with the tendency to restore their consumed power in the time frame of a second i.e. in the short term time scale. Such components are mainly induction motors and electronically controlled loads, including HVDC interconnections. In the short term timescale there is not a clear cut separation between load-driven and generatordriven stability problems.

The Voltage problem is load-driven. The voltage stability may be divided into short and long term voltage stability according to the timescale of load component dynamics. Short term voltage stability is characterized by components such as induction motors, excitation of synchronous generators and electronically controlled devices such as HVDC and static Var compensator.

When short term dynamics have died out sometime after the disturbance, the system enters a slower time frame. The dynamics of the long term scale last for several minutes. Two types of stability problems in the long term time scale are frequency and voltage problems. Frequency problems may appear after a major disturbance resulting in power system 
islanding frequency instability is related to the active power imbalance between generators and loads.

The long term voltage stability is characterized by scenarios such as load recovery by the action of on load tap changer or through load self restoration delayed control actions such as shunt compensation switching or load shedding. The modeling of long term voltage stability requires consideration of transformer on load tap changers, characteristics of static loads, manual control action of operators and automatic generation control.

\section{LOAD MODELING}

Load modeling is a difficult problem because power system loads are aggregates of many different devices. The problem is the identification of load composition at a given time and the modeling of the aggregate.

\section{Load Characteristics}

A load characteristics is an expression giving the active power or reactive power consumed by the load as a function of voltage as well as of an independent variable.

The general form of load characteristics is given by

$$
\begin{aligned}
& P=P(Z, V)--- \\
& Q=Q(Z, V)----
\end{aligned}
$$

where $\mathrm{z}$ - load demand

There are two types of loads namely exponential and polynomial loads. A widely used load characteristic is the well known exponential load which has the form

$$
\begin{aligned}
& P=Z P_{O}\left(\frac{V}{V_{O}}\right)^{\alpha}--- \\
& Q=Z Q_{O}\left(\frac{V}{V_{O}}\right)^{\beta}---
\end{aligned}
$$

where Z - Demand variable

$\mathrm{V}_{\mathrm{O}}$ - Reference voltage

$\alpha, \beta$ - Type of load

Po, Qo - Active \& reactive power

\section{Polynomial Load}

Load representation is based on summing up load components which have the same exponent. When the exponents are all integer the characteristics becomes a polynomial in V. A special case is the ZIP model, which is made up of three components namely constant impedance, constant current and constant power. The real and reactive characteristics of the ZIP load model are given by the following quadratic expressions: 


$$
\begin{aligned}
& P=a_{p}\left(\frac{V}{V_{O}}\right)^{2}+b_{p}\left(\frac{V}{V_{O}}\right)+c_{p} \\
& Q=a_{q}\left(\frac{V}{V_{O}}\right)^{2}+b_{q}\left(\frac{V}{V_{O}}\right)+c_{q}
\end{aligned}
$$

Where $\mathrm{ZP}_{\mathrm{O}}, \mathrm{ZQ}_{\mathrm{O}}$ - load real \& reactive power consumed at the Reference voltage

The types of load applied for load modeling are

- $\quad$ Voltage dependent load

- $\quad$ ZIP Load

\section{Voltage Dependent Load}

Voltage Dependent Load (VDL) are the loads whose powers are monomial functions of the bus voltage as follows

$$
\begin{aligned}
& P=P_{0}\left(\frac{V}{V_{0}}\right)^{\alpha_{p}} \\
& Q=Q_{0}\left(\frac{V}{V_{0}}\right)^{\beta}----
\end{aligned}
$$

where $\mathrm{V}_{0}$ is the initial voltage at the load bus as obtained by the power flow solution.

\section{ZIP Load}

The polynomial or ZIP loads are loads whose powers are quadratic function of the bus voltage as follows:

$$
\begin{aligned}
& P=g\left(\frac{V}{V_{0}}\right)^{2}+I_{p}\left(\frac{V}{V_{0}}\right)+P_{n}-\cdots \\
& Q=b\left(\frac{V}{V 0}\right)^{2}+I_{p}\left(\frac{V}{V_{0}}\right)+Q_{n}-\cdots
\end{aligned}
$$

Where $\mathrm{V}_{0}$ is the initial voltage at the load bus as obtained by the power flow solution.

\section{IV.MODAL ANALYSIS}

Stability is determined by computing the V-P and Q-V curves at the selected load buses. Generally, such curves are generated by executing a large number of power flows using conventional models. While such procedures can be automated, they are time-consuming and do not readily provide information useful in gaining insight into causes of stability problems. In addition, these procedures focus on individual buses, that is the stability characteristics are established by stressing each bus independently. This may distort the stability condition of the system. The modal analysis approach provides the information regarding the mechanism of instability.

The network constraints can be represented in the following linearized form. 


$$
\left[\begin{array}{c}
\Delta P \\
\Delta Q
\end{array}\right]=\left[\begin{array}{l}
J_{P \theta} J_{P v} \\
J_{Q \theta} J_{Q v}
\end{array}\right]\left[\begin{array}{c}
\Delta \theta \\
\Delta v
\end{array}\right]
$$

Where

$$
\begin{aligned}
& \Delta P=\text { Incremental change in bus real power } \\
& \Delta Q=\text { Incremental change in bus reactive power injection } \\
& \Delta \theta=\text { Incremental change in bus voltage angle } \\
& \Delta v=\text { Incremental change in bus voltage magnitude }
\end{aligned}
$$

The elements of Jacobian matrix gives the sensitivity between power flow and bus voltage changes.

System voltage stability is affected by both $\mathrm{P}$ and $\mathrm{Q}$. However at each operating point we may keep P constant and evaluate voltage stability by considering the incremental relationship between $\mathrm{Q}$ and $\mathrm{V}$. Based on the above considerations, let $\Delta P=0$. Then

$$
\begin{aligned}
& \Delta Q=J_{R} \Delta V \\
& \text { Where } J_{R}=\left\lfloor J_{Q V}-J_{Q \theta} J_{P \theta}^{-1} J_{P V}\right\rfloor--
\end{aligned}
$$

and $J_{R}$ is the reduced Jacobian matrix of the system. From equation 11, we may write

$$
\Delta V=J_{R}^{-1} \Delta Q
$$

The V-Q Sensitivity at a bus represents the slope of the Q-V curve at the given operating point. A positive V-Q sensitivity is indicative of stable operation. The smaller the sensitivity, the more stable the system. As stability decreases, the magnitude of the sensitivity increases, becoming infinite at the stability limit. Conversely, a negative V-Q sensitivity is indicative of unstable operation. A small negative sensitivity represents a very unstable operation. Because of the nonlinear nature of $\mathrm{V}-\mathrm{Q}$ relationships, the magnitudes of the sensitivities for different system conditions do not provide a direct measure of the relative degree of stability.

\section{Q-V Modal Analysis}

$$
\text { Let } J_{R}=\xi \wedge \eta
$$

Where $\xi=$ right eigenvector matrix of $\mathrm{J}_{\mathrm{R}}$

$\eta$ = left eigenvector matrix of $\mathrm{J}_{\mathrm{R}}$

$\wedge=$ diagnol eigenvalue matrix of $J_{R}$

From the equation 14, 
$J_{R}^{-1}=\xi \wedge^{-1} \eta$

Substituting in the equation 13 gives

$$
\begin{aligned}
& \Delta V=\xi \wedge^{-1} \eta \Delta Q \\
& \Delta V=\sum_{i} \frac{\xi_{i} \eta_{i}}{\lambda_{i}} \Delta Q
\end{aligned}
$$

Where $\xi_{i}$ is the $\mathrm{i}^{\text {th }}$ column right eigenvector and $\eta_{i}$ is the $\mathrm{i}^{\text {th }}$ row left eigenvector of $\mathrm{J}_{\mathrm{R}}$.

Each eigenvalue $\lambda_{i}$ and the corresponding right and left eigenvectors $\xi_{i}$ and $\eta_{i}$ define the $\mathrm{i}^{\text {th }}$ mode of $\mathrm{Q}-\mathrm{V}$ response.

\section{ANFIS}

The abbreviation for ANFIS is adaptive neuro fuzzy inference system. It maps inputs through input membership functions and associated parameters, and then through output membership functions and associated parameters to outputs, can be used to interpret the input/output map. The input vector considered is power and the output vector is Voltage.

This is the major training routine for Sugeno-type fuzzy inference systems. Anfis uses a hybrid learning algorithm to identify parameters of Sugeno-type fuzzy inference systems. It applies a combination of the least-squares method and the back propagation gradient descent method for training FIS membership function parameters to emulate a given training data set.

\section{ALGORITHM}

- $\quad$ Step 1: Consider a sample six bus system

- Step 2: Using newton-raphson method, calculate the voltage of the load buses and this voltage as a reference voltage

- Step 3: Model for voltage dependent load and calculate the real and reactive power

- $\quad$ Step 4: Model for ZIP load and calculate the real and reactive power

- $\quad$ Step 5: Follow the same procedure for 30 bus system

\section{Algorithm for Modal Analysis}

- $\quad$ Step 1: Consider a 2 bus system

- Step 2: Using newton- raphson method, calculate the voltage and angle for the load buses

$$
\begin{aligned}
& P_{k}=V_{k} \sum_{m=1}^{n}\left(G_{k m} V_{m} \cos \theta_{k m}+B_{k m} V_{m} \sin \theta_{k m}\right) \\
& Q_{k}=V_{k} \sum_{m=1}^{n}\left(G_{k m} V_{m} \sin \theta_{k m}-B_{k m} V_{m} \cos \theta_{k m}\right)
\end{aligned}
$$


- Step 3: Using the power flow equation for modal analysis, calculate the jacobian expressions using the equation 6.2

$$
\begin{aligned}
& J_{p \theta}=-G_{k m} V_{m} \sin \theta_{k m}+B_{k m} V m \cos \theta_{k m} \\
& J_{p v}=G_{k m} \cos \theta_{k m}+B_{k m} \sin \theta_{k m}+2 V_{m} \\
& J_{q \theta}=G_{k m} V_{m} \cos \theta_{k m}+B_{k m} V_{m} \sin \theta_{k m} \\
& J_{q v}=G_{k m} \sin \theta_{k m}-B_{k m} \cos \theta_{k m}+2 V_{m} \\
& J_{R}=J_{q v}-J_{q \theta} *\left[J_{p \theta}\right]^{-1} * J_{p v}
\end{aligned}
$$

- Step 4: Substitute the voltage and angle of the load buses in the jacobian expressions using the equation 19 and calculate the reduced jacobian matrix using the equation 20

- Step 5: Repeat the same procedure for six bus system

\section{Algorithm for Load Modeling using Anfis}

- Step 1: Consider a 6 bus system.

- Step 2: Using NR method, calculate the voltage and keep this voltage as reference voltage.

- Step 3: Model for voltage dependent load and ZIP load and calculate the real \& reactive power.

- Step 4: Train the system using ANFIS \& substitute the voltage in the corresponding load equations and calculate the real \& reactive power.

- Step 5: Error is calculated between normal load modeling and using ANFIS.

- Step 6: Procedure is repeated for 30 bus system.

\section{RESULTS AND DISCUSSIONS}

The loads applied for load modeling are voltage dependent load and ZIP load. The system considered for load modeling are 6 bus system and 30 bus system. Figure shows the one line diagram of the six bus system and 30 bus system.

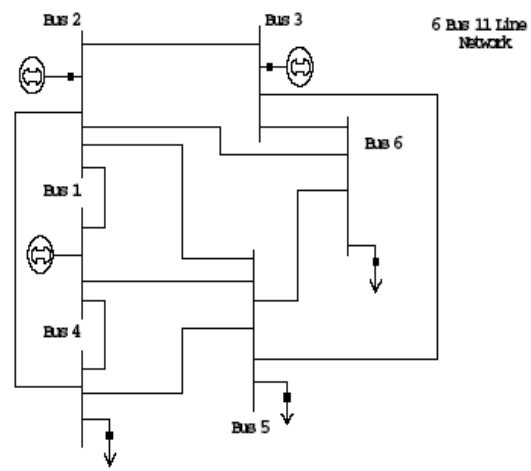

Figure 1 
Initially, for the six bus system load modeling is carried out with voltage dependent load and ZIP load and its corresponding outputs are obtained. Then for the 30 bus system, load modeling is carried out for the voltage dependent load and ZIP load. For each type of the load, the load is gradually increased by 5\%, 10\% and 15\% and its corresponding outputs are obtained. Again for the six bus system and 30 bus system load modeling is carried out for voltage dependent load and ZIP load and its corresponding outputs are obtained

\section{Modal Analysis}

Modal analysis provides the information regarding the mechanism of instability. Modal analysis is carried out six bus system. Through Modal analysis, we can determine the weakest bus in the system by gradually increasing the load. This gives the opportunity to introduce the facts devices in order to improve the stability of the system. Through modal analysis, for a particular value of load, we can determine the jacobian matrix. By gradually increasing the load, we can also determine the jacobian matrix for the load buses. Whenever the jacobian value goes to zero for a particular load bus, we can say that the particular bus is the weakest bus in the system.

\section{Load Modeling using Anfis}

The 6 bus system is trained using ANFIS (Adaptive neuro- fuzzy inference system) and ANFIS ouput voltage is substituted in the corresponding voltage dependent load and ZIP load equations to calculate P and Q. Error is calculated between normal load modeling and using an fis. For each type of the load, the load is gradually increased by 5\%, 10\%, $11 \%$ and $13 \%$ and its corresponding outputs are obtained.

Table 1: Voltage Dependent Load for Six Bus System

\begin{tabular}{|c|c|c|c|c|c|c|c|c|}
\hline \multirow{2}{*}{ S. No } & \multirow{2}{*}{ Po } & \multirow{2}{*}{ Qo } & \multicolumn{2}{c|}{ Load Model } & \multicolumn{2}{c|}{ Anfis } & \multicolumn{2}{c|}{ Error } \\
\hline & & & $\mathbf{P}$ & $\mathbf{Q}$ & $\mathbf{P}$ & $\mathbf{Q}$ & $\mathbf{P}$ & $\mathbf{Q}$ \\
\hline 1 & 0.75 & 1.000 & 0.7488 & 0.9984 & 0.7497 & 0.9997 & 0.0009 & 0.0013 \\
\hline 2 & 0.85 & 1.133 & 0.8460 & 1.1277 & 0.8500 & 1.1330 & 0.0040 & 0.0053 \\
\hline 3 & 0.90 & 1.200 & 0.8943 & 1.1924 & 0.800 & 1.2000 & 0.0057 & 0.0076 \\
\hline 4 & 1.10 & 1.466 & 1.0850 & 1.4461 & 1.100 & 1.4667 & 0.0155 & 0.0206 \\
\hline 5 & 1.30 & 1.733 & 1.2710 & 1.6943 & 1.300 & 1.7329 & 0.0290 & 0.0386 \\
\hline 6 & 1.50 & 2.000 & 1.4497 & 1.9324 & 1.500 & 2.000 & 0.0503 & 0.0676 \\
\hline 7 & 1.70 & 2.266 & 1.6127 & 2.1491 & 1.700 & 2.266 & 0.0685 & 0.1169 \\
\hline
\end{tabular}

Table 2: ZIP Load for Six Bus System

\begin{tabular}{|c|c|c|c|c|c|c|c|c|}
\hline \multirow{2}{*}{ S. No } & \multirow{2}{*}{ Po } & \multirow{2}{*}{ Qo } & \multicolumn{2}{c|}{ Load Model } & \multicolumn{2}{c|}{ Anfis } & \multicolumn{2}{c|}{ Error } \\
\hline & & & P & Q & P & Q & P & Q \\
\hline 1 & 0.75 & 1.000 & 0.7180 & 0.8769 & 0.7222 & 0.8815 & 0.0042 & 0.0046 \\
\hline 2 & 0.85 & 1.133 & 0.8224 & 1.0182 & 0.8406 & 1.038 & 0.0182 & 0.0206 \\
\hline 3 & 0.90 & 1.200 & 0.8746 & 1.0894 & 0.9012 & 1.1199 & 0.0266 & 0.0305 \\
\hline 4 & 1.10 & 1.466 & 1.0836 & 1.3727 & 1.1594 & 1.463 & 0.0758 & 0.0903 \\
\hline 5 & 1.30 & 1.733 & 1.2930 & 1.6581 & 1.4421 & 1.8467 & 0.1491 & 0.3986 \\
\hline 6 & 1.50 & 2.000 & 1.5031 & 1.9454 & 1.776 & 2.2867 & 0.2729 & 0.3413 \\
\hline 7 & 1.70 & 2.266 & 1.7148 & 2.2364 & 2.2264 & 2.8859 & 0.5116 & 0.6495 \\
\hline
\end{tabular}

\section{Bus System}

The 30 bus system is trained using ANFIS (Adaptive neuro- fuzzy inference system) and ANFIS ouput voltage is substituted in the corresponding voltage dependent load and ZIP load equations to calculate P and Q. Error is calculated between normal load modeling and using anfis. For each type of the load, the load is gradually increased by $5 \%, 10 \%, 11 \%$ and $13 \%$ and its corresponding outputs are obtained. 
Table 3: Voltage Dependent Load for 30 Bus System

\begin{tabular}{|c|c|c|c|c|c|c|}
\hline \multirow{2}{*}{$\%$} & \multicolumn{2}{|c|}{ Load Model } & \multicolumn{2}{c|}{ ANFIS } & \multicolumn{2}{c|}{ Error } \\
\cline { 2 - 7 } & $\mathbf{P}$ & $\mathbf{Q}$ & $\mathbf{P}$ & $\mathbf{Q}$ & $\mathbf{P}$ & $\mathbf{Q}$ \\
\hline $5 \%$ & 0.1172 & 0.0892 & 0.1189 & 0.1577 & 0.0017 & 0.0685 \\
\hline $10 \%$ & 0.1640 & 0.2184 & 0.1683 & 0.2243 & 0.0043 & 0.0059 \\
\hline $11 \%$ & 0.1729 & 0.2302 & 0.1785 & 0.2376 & 0.0056 & 0.0074 \\
\hline $13 \%$ & 0.1819 & 0.2422 & 0.1925 & 0.2559 & 0.0104 & 0.0137 \\
\hline
\end{tabular}

Table 4: ZIP Load for 30 Bus System

\begin{tabular}{|c|c|c|c|c|c|c|}
\hline \multirow{2}{*}{$\%$} & \multicolumn{2}{|c|}{ Load Model } & \multicolumn{2}{c|}{ ANFIS } & \multicolumn{2}{c|}{ Error } \\
\cline { 2 - 7 } & $\mathbf{P}$ & $\mathbf{Q}$ & $\mathbf{P}$ & $\mathbf{Q}$ & $\mathbf{P}$ & $\mathbf{Q}$ \\
\hline $5 \%$ & 0.0771 & 0.1364 & 0.0813 & 0.1456 & 0.0042 & 0.0092 \\
\hline $10 \%$ & 0.1277 & 0.1548 & 0.1396 & 0.1777 & 0.0119 & 0.0229 \\
\hline $11 \%$ & 0.1388 & 0.1588 & 0.1581 & 0.1877 & 0.0193 & 0.0289 \\
\hline $13 \%$ & 0.1626 & 0.1817 & 0.200 & 0.222 & 0.0374 & 0.0403 \\
\hline
\end{tabular}

\section{CONCLUSIONS}

Modeling of voltage dependent load and ZIP load is carried out in order to predict the maximum deliverable power. Initially normal load modeling is done using the corresponding load equations, then the real power, reactive power and the voltage of the corresponding load buses is trained using Adoptive Neuro Fuzzy Inference System (ANFIS). The voltage of the corresponding load buses is obtained using ANFIS. By substituting these corresponding voltages in the corresponding load equations, we can obtain the real and reactive power. Now Error is calculated between the normal load modeling and ANFIS load modeling. Advantages of ANFIS is that, it is computationally efficient. It works well with linear techniques (e.g., PID control). It works well with optimization and adaptive techniques. It has guaranteed continuity of the output surface. It is well-suited to mathematical analysis.

\section{REFERENCES}

1. “IEEE Task Force on load representation for dynamic Performance”, IEEE Transactions on power systems, Vol 10, No 3, August 1995.

2. David J.Hill, "Non - linear Dynamic Load models with recovery for voltage stability studies", IEEE transactions on power systems, Vol 8, No 1, February 1993.

3. Ahmad, M. Mustafaiz, et al. "Optimization of Process Parameters in Electric Discharge Machining Process." International Journal of Mechanical Engineering (IJME) (2016).

4. William.W.Price, "Load modeling for power flow and Transient stability computer studies", IEEE transactions on power systems, Vol3”, No 1, February 1998.

5. Gia-TongVuong, "Modeling a mixed Residential-Commercial Load for simulations involving Large disturbances", IEEE transactions on power systems, Vol 12", No 2, May 1997.

6. Pande, Pravin P., and R. K. Sambhe. "Artificial Intelligence Machining Predictions by Fuzzy GUI For Inconel 718 with PVD Coated Carbide Cutting Tool." International Journal of Mechanical and Production Engineering Research and Development (IMPERD) 7.3 (2017): 313-320.

7. Daniel Morrison, "Modeling and identification of non-linear dynamic loads in power systems", IEEE transactions on power systems, Vol 9", No 1, February 1994 
8. M.K.Pal, "Voltage stability conditions considering load characteristics", IEEE transactions on power systems, Vol7”, No 1, February 1992.

9. Hemachandra, S., and R. V. S. Satyanarayana. "Co-active neuro-fuzzy inference system for prediction of electric load." IJEEER 3.2 (2013): 217-222.

10. J.R.Shin, "Improvement of PV curve considering the effects of voltage dependent load models and transmission losses for stability analysis”, IEE Proceeding - gener, transm, Distr, Vol 149, No 4, July 2002.

11. X.Gu and C.A.Canizares, "Fast prediction of loadability margins using neural networks to approximate security boundaries of power systems", IET Gener.Trans.Distr, 2007, pp.466-475.

12. Babu, N., SM Vinu Kumar, and A. Megalingam Murugan. "Multi-Response Optimization of Process Parameters in Wire Electric Discharge Machining of Ti-3al-2.5 V Alloy using Taguchi Integrated Grey Relational Analysis. " International Journal of Mechanical and Production Engineering Research and Development (IJMPERD) 9. 1, Jan 2019, 233-242

13. Thierry Van Cutsem, "Voltage Stability of Electric power system”, kluwer academic publishers.

14. Kip Morison, “Load Modeling for voltage stability studies”, IEEE Transactions on power systems, 2006.

15. P.Kundur, Power system stability and control, McGraw-Hill, 1994. 